\title{
L’eSPACE et LA MÉMOIRE DANS LIEUX DE GeORges PereC
}

\author{
Jagoda KRYG \\ Université Jagellonne, Kraków
}

\begin{abstract}
En): Space is one of the most interesting motifs in the work of Georges Perec and a theme that the author has dealt with numerous times in his various novels and essays. For Perec, space is inextricably linked to the place of remembrance which keeps the memories of the past. The spaces associated with the mother and father of the author, of whom he had almost no memories left, have become for him the only places to look for the traces of the past, to find common bonds and to fill the void left by the war. What characterizes Perec's writing is the use of different strategies aimed at saving space from oblivion through the meticulous recording of its progressive changes and the establishment of their various classifications. The objective of the article is to study how the spaces in Georges Perec's work can be linked to the remembrance or the creation of memories and also how they represent the absence, the inability of the memory and the void that is impossible to fil.
\end{abstract}

Keywords (En): Perec, space, memory, loss, OuLiPo.

Mots-clés (Fr) : Perec, espace, mémoire, perte, OuLiPo.

L'espace est un doute : il me faut sans cesse le marquer, le désigner ; il n'est jamais à moi, il ne m'est jamais donné, il faut que j'en fasse la conquête.

Georges PEREC (2012: 179)

Lorsqu'on réfléchit sur les fascinations les plus manifestes dans l'œuvre de Georges Perec, celle de l'espace nous vient à l'esprit de manière presque instantanée. Tentative d'épuisement d'un lieu parisien, Lieux ou Espèces d'espaces, sont seulement quelques titres qui nous permettent déjà de remarquer une certaine dominante thématique. Et bien que les œuvres les plus importantes inscrites dans ce courant aient été écrites par Perec dans la seconde moitié des années 1970, la question de l'espace, et plus précisément celle concernant le lien entre l'espace et la mémoire, occupait l'écrivain depuis longtemps, comme attesteatteste une lettre à Maurice Nadeau envoyée en 1969 dans laquelle l'auteur parle de plusieurs projets littéraires concernant ce sujet dans lesquels il voulait s'engager'.

L'attention portée au problème de l'espace, apparente dans les formes romanesques aussi bien que dans les essais, s'est manifestée chez Perec de façons assez différentes. La rhétorique de l'infra-ordinaire est particulièrement visible dans divers types de descriptions et classifications littéraires, y compris celles de l'espace. Ces deux techniques ont été employées, par exemple, dans Espèces d'espace, une sorte de répertoire des lieux : du plus petit au plus grand. Une autre

\footnotetext{
${ }^{1}$ Voir : Perec, G. (1990) Lettre à Maurice Nadeau (7 juillet 1969). Je suis né, Paris, Éditions du Seuil, 51-66.
} 
approche, adoptée dans Tentative d'épuisement d'un lieu parisien, qui exigeait encore plus de travail descriptif et minutieux, visait à décrire la Place Saint-Sulpice pendant trois jours consécutifs passés sur place à différents moments de la journée. En d'autres termes, Perec voulait épuiser cet endroit en perpétuant par écrit son image quotidienne et transitoire.

Enfin, l'espace perecquien est aussi lié aux notions de mémoire et de souvenir. Pourtant, en ce qui concerne l'œuvre de Perec, ce n'est pas seulement la mémoire qui garde le souvenir d'un lieu, mais, premièrement, c'est l'espace qui constitue pour l'auteur un immense réservoir d'histoires et une potentialité mnémonique.

\section{Maîtrise de la mémoire}

L'obsession classificatoire, la précision du détail et la peur d'oublier ne sont néanmoins pas, comme nous pourrions le supposer, les résultats uniques de la rhétorique de l'infra-ordinaire ou de la contrainte oulipienne. Comme le remarque Perec dans Les lieux d'une ruse, son anxiété, qui s'exprimait par le désir d'apprivoiser les objets et l'espace environnants, avait un rapport direct avec la psychanalyse qu'il entreprenait au début des années 1970 avec Jean-Bertrand Pontalis :

En même temps [lorsqu'il a commencé la psychanalyse] s'instaura comme une faillite de ma mémoire : je me suis mis à avoir peur d'oublier, comme si, à moins de tout noter, je n'allais rien pouvoir retenir de la vie qui s'enfuyait. Chaque soir, scrupuleusement, avec une conscience maniaque, je me mis à tenir une espèce de journal : c'était tout le contraire d'un journal intime ; je n'y consignais que ce qui m'était arrivé « d'objectif » : l'heure de mon réveil, l'emploi de mon temps, mes déplacements, mes achats, le progrès - évalué en quelques lignes ou en pages - de mon travail (...). Cette panique de perdre mes traces s'accompagna d'une fureur de conserver et de classer (PEREC, 1985 : 69).

Grâce à la psychothérapie, Perec a donc commencé à questionner son passé et à chercher ce qu'il croyait être caché dans les recoins de sa mémoire. Pourtant, comme son investigation avançait, sa mémoire se montrait toujours plus élusive et ses souvenirs d'enfance fragmentaires et incomplets. Ayant réalisé ce défaut de la mémoire et le fait qu'au fil du temps elle devient, en réalité, plus susceptible aux influences et aux modifications subconscientes, Perec s'est lancé dans la recherche d'autres moyens qui pouvaient l'aider à maîtriser ses souvenirs. D'ailleurs, ses capacités développées dans ce domaine ont impressionné son psychothérapeute qui, ayant reconnu que «les souvenirs d'enfance (...) n'étaient pas son fort » (BELLOS, 1994 : 538), a également noté que « sa mémoire était immense, prête à accueillir non : à enregistrer - toutes sortes d'informations » (ibidem; c'est nous qui soulignons). Aussi, c'est pendant les dernières années de la psychanalyse que Perec a écrit $W$ ou le souvenir d'enfance, l'un de ses textes majeurs concernant tout ce dont l'auteur pouvait se souvenir des premières années de sa vie. À partir de ce moment, il a commencé à accorder plus d'attention aux liens entre la mémoire et l'espace - et comme le remarque Jean-Bertrand Pontalis, c'est Perec qui lui a finalement fait « apercevoir ce que peut signifier la passion des lieux » (ibidem).

Toutefois, cette volonté obsessionnelle d'enregistrer le présent quotidien et de se remémorer les premières années de sa vie, que Perec affirme avoir presque 
oubliées ${ }^{2}$, trouve sa source non seulement dans le travail de psychanalyse, mais résulte aussi d'une révolte contre la suppression de la mémoire à laquelle l' auteur a dû se soumettre pendant la guerre en dissimulant - et finalement en oubliant - sa judéité et son origine. La nécessité de créer des archives mnémoniques avec une précision presque mathématique peut donc être perçue comme un résultat de cette suppression.

\section{L’espace comme réservoir de la mémoire}

Le premier et l'un des projets les plus ambitieux de Perec, liés à la mémoire de l'espace, est Lieux, une œuvre inachevée que l'auteur a abandonnée au bout de six ans de travail. En 1969, Perec a choisi douze lieux à Paris qu'il voulait décrire pendant douze ans. Chaque mois, il travaillait sur deux endroits parisiens : la première description était produite sur place et de la manière la plus exhaustive possible, tandis que la seconde était faite de mémoire et consistait plutôt à évoquer tous les souvenirs et associations qui étaient liés à chaque endroit. Le procédé devait se répéter chaque année et continuer de la même manière pendant douze ans, tandis que chaque description, une fois écrite, était mise sous enveloppe scellée pour ne pas influencer les observations ultérieures. L'objectif de Perec présupposait 288 enveloppes en totalité, mais le travail abandonné en 1975 a laissé l'auteur avec 133 textes dont plusieurs ont été utilisés pour d'autres projets. Selon le plan initial, l'écrivain voulait montrer le vieillissement simultané des lieux, de l'écriture et des souvenirs (PEREC, 1985 : 59). Les douze lieux n'ont pas d'ailleurs été choisis de manière arbitraire. Chacun d'entre eux correspondait à un certain moment de la vie de l'auteur. On peut donc parler de ces endroits comme des lieux de mémoire dont Perec voulait non seulement sauver le souvenir, mais aussi le fabriquer sur place, pour que leur image, telle qu'il la décrivait de manière objective, ne s'efface jamais de la mémoire collective.

Ainsi, la première étape de cette écriture, qui consistait en une description précise et neutre, visait à créer une sorte de mémoire des lieux pour témoigner de leur transformation lente et imperceptible au quotidien. Ces observations ne cherchaient pas cependant à produire une mythologie de l'espace, ne mettant pas en valeur leur importance subjective ou historique, mais elles constituaient plutôt des enregistrements de la banalité, donc de ce qui se montre le plus souvent comme insignifiant. La poursuite de la quotidienneté, enchainée de manière évidente à la rhétorique de l'infra-ordinaire, que Perec appelait aussi «le bruit de fond », était, par conséquent, sa manière de lutter contre l'oubli accablant. Selon Maurice Blanchot, « le quotidien est ce qu'il y a de plus difficile à découvrir » (BLANCHOT, 1969 : 335). Suivant cette idée, Perec a noté que notre perception obscurcit souvent ce qui, d'après lui, détermine l'essence de la vie :

\footnotetext{
${ }^{2}$ L'auteur commence $W$ ou le Souvenir d'enfance comme suit : «Je n'ai pas de souvenir d'enfance. Jusqu'à ma douzième année à peu près, mon histoire tient en quelques lignes : j'ai perdu mon père à quatre ans, ma mère à six ; j'ai passé la guerre dans diverses pensions de Villard-de-Lans. En 1945, la sœur de mon père et son mari m'adoptèrent » (PEREC, $1975: 13$ ).
} 
Ce qui se passe vraiment, ce que nous vivons, le reste, tout le reste, où est-il ? Ce qui se passe chaque jour et qui revient chaque jour, le banal, le quotidien, l'évident, le commun, l'ordinaire, l'infra-ordinaire, le bruit de fond, l'habituel, comment en rendre compte, comment l'interroger, comment le décrire ? Interroger l'habituel (...). Nous dormons notre vie d'un sommeil sans rêves. Mais où est-elle, notre vie ? Où est notre corps ? Où est notre espace ? (PEREC, 1989 : 11)

Voilà l'objectif de cette écriture que Philippe Lejeune va désigner comme une forme particulière de l'autobiographie : capturer la vie dans toute sa simplicité, dans la circulation routière ou dans les mouvements des pigeons qui marchent sur les pavés. C'est une lutte contre l'amnésie, mais aussi un moyen de marquer l'espace, de s'en servir pour se définir soi-même et reconquérir son identité perdue. Selon Perec, c'est l'ordinaire qui nous détermine : où nous vivons, ce qui se trouve sur notre bureau ou la route que nous prenons d'habitude pour nous rendre chez l'épicier. Nous marquons notre espace avec nos empreintes subtiles et constantes, nous nous l'approprions, mais, en même temps, nous nous inscrivons également dans son histoire plus vaste. À travers la description des lieux parisiens, Perec crée, en effet, un large amalgame de perceptions communes, de petits lambeaux de l'ordinaire appartenant à ce qu'on peut appeler la mémoire globale, semblable à celle dont l'auteur fait preuve dans Je me souviens.

\section{Le nom qui confirme l'existence}

L'impératif de définir, d'énumérer et de cataloguer - non seulement l'espace, mais aussi les objets et les pensées - constituait, de plus, une expérience phénoménologique et primaire, celle du retour aux sources et de la recherche de l'origine, d'un élément manquant. Comme le constate Maryline Heck, «ce qui se passe devant [1] es yeux est une manière aussi de se donner un ancrage dans le réel, de situer son corps dans l'espace environnant» (HECK, 2012: 68). Enfin, nommer c'est confirmer sa propre existence. Mais avant de se situer dans "l'espace environnant», il faut d'abord retourner à une expérience fondamentale de l'observation et regarder comment les morceaux de l'espace émergent. La description neutre, nous pourrions même dire indifférente, concentrée uniquement sur ce qui est objectivement disponible pour les yeux, peut être envisagée comme un réalisme du regard et du langage qui n'est pas obscurci par la métaphore, ni impliqué dans un réseau d'associations libres. Les descriptions protocolaires des lieux, comme celles de la Place d'Italie, de l' ̂lle Saint-Louis ou de l'Avenue Junot, donc des endroits familiers à chaque habitant de Paris, constituent, en réalité, une tentative de perception renouvelée. Cependant, notre vision du quotidien devient d'habitude distordue à cause des images et des souvenirs qui s'y superposent et qui empêchent, par conséquent, d'atteindre ce qui est l'essence des lieux, une sorte d'évidence individuelle.

Dans l'œuvre littéraire de Perec, la rue Vilin, l'un des douze endroits choisis pour Lieux, sert de point de référence singulier et du lieu de mémoire le plus important. C'est là, au numéro 24 , que l'écrivain a passé les six premières années de son enfance et où sont nés les seuls souvenirs qu'il a gardés de ses parents. Quand en 1975 Perec finit sa psychanalyse, il renonce également à l'écriture de Lieux, mais 
les enveloppes avec les descriptions de la rue Vilin seront exceptionnellement ouvertes et leur contenu inclus dans le recueil L'Infra-ordinaire.

Comme Perec l'écrit dans la partie autobiographique du roman $W$ ou le souvenir d'enfance, dans la période entre la guerre et le début de son travail sur Lieux, il a revu la rue Vilin seulement deux fois (PEREC, 1975 : 72). La première avait un rapport avec une visite assez brève chez sa grand-mère, peu après la fin de la guerre. La deuxième s'est passée à l'occasion d'une promenade avec des amis dans les années 1960 pendant laquelle « [1]a rue n'évoqua en [lui] aucun souvenir précis, à peine la sensation d'une familiarité possible » (ibidem). Ce n'est donc que lors de son travail littéraire sur ce projet que l'auteur a ressenti, et transmis dans son écriture, l'expérience directe et particulière de la mort d'un lieu. Chaque année Perec observait et décrivait alors la disparition progressive du paysage de son enfance : la démolition successive et inéluctable des bâtiments, l'écroulement des murs des maisons abandonnées ou l'effacement de la peinture écaillée de l'inscription « COIFFEUR DAMES », l'endroit où travaillait sa mère. Philippe Lejeune prétend que «Lieux est le tombeau d'un amour (...) le lieu d'un combat entre la vie et la mort ». Il remarque aussi qu" "[i]l se passe pour Lieux la même chose que pour La Disparition : complémentarité entre le jeu de la contrainte et le travail de deuil » (LEJEUNE, 1991 : 158). C'est, en réalité, La rue Vilin qui est l'un des récits littéraires les plus poignants qui parlent de la mort et de la disparition. Parmi d'autres textes écrits pour Lieux, La rue Vilin prend une forme particulière, ressemblant un peu à une sorte d'adieu définitif à un espace, la dernière liaison entre l'auteur et ses parents, une preuve matérielle de leur existence et de son identité perdue.

En observant ses retours annuels au numéro 24, à l'immeuble qui subsistait encore intact malgré la guerre et le temps passé, Perec démontre que les lieux gardent les souvenirs beaucoup plus longtemps que les hommes, mais quand ils disparaissent, avec eux se dissipent également les dernières traces de l'existence humaine. Entre février 1969 et septembre 1975, Perec essaie alors d'archiver, avec la précision qui lui est propre, les derniers moments de la vie de la rue dont il pressent le délabrement imminent, et, par conséquent, celui du dernier lien entre le passé et le présent.

Jeudi 25 juin 1970

(...) $\mathrm{Au} 24$, dans la courette, il y a un chat sur une soute à charbon. L'inscription COIFFURE DAMES est encore visible.

Mercredi 13 janvier 1971

(...) Au 24 : coiffure dames (pas le magasin, seulement la trace de l'enseigne peinte sur le mur ; dans la cour du 24, des poutrelles de métal ; des ouvriers en face réparent un toit (d'un immeuble rue des Couronnes?) Au loin des grues.

Mercredi 13 janvier 1971

(...) 24 toujours intact, 25 : un magasin fermé ; 26 : des fenêtres murées, 27 muré, 28, 30, 36 toujours debout. Un chat tigré et un chat noir dans la cour du 24.

Jeudi 21 novembre 1974,

(... ) Le 18 et le 22 sont des cafés hôtels encore debout, ainsi que le 20 et le 24. Du côté impair, le 21 est en démolition (on voit des bulldozers, des excavatrices, de feux), le 23 et le 25 sont éventrés. Après le 25 plus rien. 


\section{7 septembre 1975}

La quasi-totalité du côté impair est couverte de palissades en ciment. Sur l'une d'elles un graffiti : TRAVAIL = TORTURE $($ PEREC, $1989: 23-31)$.

Les descriptions, faites sur place, conformément à la règle adoptée, constituent donc un enregistrement de ce processus graduel d'anéantissement. Pourtant, selon Pierre Getzler, qui accompagnait l'auteur à la rue Vilin comme photographe, Perec y écrivait toujours de la position d'un «usager de l'espace », en mouvement constant (GETZLER ; DEPAULE, 2012 : 85-87), comme s'il voulait réanimer, encore une fois, ce paysage agonisant. L'image de la rue Vilin gardée par Perec montre que le quotidien, cette banalité que nous trouvons tellement monotone est, en fait, soumis à un processus de modifications lentes mais stables. Chaque jour il se produit dans la réalité de l'ordinaire des micro-changements, imperceptibles pour les yeux, mais qui se rendent cependant évidents pendant une analyse longue et perspicace, comme celle qui est effectuée dans Lieux. Ce désir perecquien d'une réalité parfaite - constante et fixe - comme celle de la photographie, se manifestera, par ailleurs, dans toute la création littéraire de l'auteur, le plus visiblement dans Espèces d'espaces :

J'aimerais qu'il existe des lieux stables, immobiles, intangibles, intouchés et presque intouchables, immuables, enracinés ; des lieux qui seraient des références, des points de départ, des sources (...). Mes espaces sont fragiles : le temps va les user, va les détruire : rien ne ressemblera plus à ce qui était, mes souvenirs me trahiront, l'oubli s'infiltrera dans ma mémoire, je regarderai sans les reconnaître quelques photos jaunies aux bords tout cassés (PEREC, 2012 : 179).

La description de la rue Vilin constitue en particulier une façon de sauver les souvenirs refoulés par la mémoire. C'est aussi une tentative de capturer le «bruit de fond », le déclin de la vie d'un espace ordinaire. Mais c'est également une interrogation timide sur les traces «d'eux » qui, peut-être, subsistent encore dans les décombres, dans les marques de la peinture ou dans la mémoire des autres, comme dans les souvenirs de la dame qui sort de l'immeuble d'à côté et « qui vit là depuis 36 ans » et « se souvient très bien de la coiffeuse du 24 » (PEREC, 1989 : 27). De la même manière, l'auteur investit l'espace de Lubartów, la ville natale de son père, lors de son voyage en Pologne en 1981. Comme le relate Claude Roy, Perec est revenu de cette visite « fourbu », sans avoir trouvé de «traces », en constatant que «tout est effacé » (ROY, 1983 : 208). Dans un entretien donné quelques jours après, il a admis : « Je n'ai pas de maison, de famille, je n'ai pas de grenier, comme on dit, je n'ai pas de racines, je ne les connais pas » (PAWLIKOWSKA, 2003 : 207). L'espace s'est montré, encore une fois, muet et impénétrable, incapable de ressusciter la mémoire longtemps oubliée.

L'approche que Perec adopte par rapport à l'espace qui est visible dans Lieux est de toute façon assez significative. Nous pouvons remarquer qu'elle ressemble un peu à une technique de lecture textuelle, à une sorte de déchiffrage. C'est l'auteur qui indique d'ailleurs que «[1]e problème n'est pas d'inventer l'espace, encore moins de le réinventer (...), mais de l'interroger, ou, plus simplement encore, de le 
lire (...) »(PEREC, 2012 : page non numérotée). Nous pouvons donc comparer Lieux, et surtout la partie La rue Vilin, à une méthode analytique de lecture pénétrante, une recherche structurelle qui vise à trouver des sens cachés. L'espace est une attente pour que le superficiel se disperse et révèle une vérité oubliée.

\section{Contre le vieillissement des souvenirs}

À part La rue Vilin, Perec a publié aussi d'autres descriptions « objectives » qui devaient faire partie de Lieux, comme celles du carrefour Mabillon, de la Place d'Italie ou de la rue de l'Assomption. Toutes ces publications étaient le résultat de la première étape, celle qui cherchait à atteindre un récit générique. Mais comme nous l'avons déjà souligné, la conception initiale de l'écrivain présupposait une écriture en deux étapes que Perec a rejetée lorsqu'il a abandonné le projet. Effectivement, l'auteur n'a ouvert aucune enveloppe contenant les textes qui étaient les résultats de cette deuxième phase de son travail, dont l'objectif était de démontrer les associations entre les lieux et les souvenirs. L'effet littéraire dont parlait initialement Perec, c'est-à-dire la mise en relief du «triple vieillissement» : de l'espace, des souvenirs et de l'écriture, n'a jamais été réalisé. La publication des fragments de Lieux, telle qu' elle se présente actuellement, ne montre que le premier aspect. Mais il serait intéressant d'observer le véritable fonctionnement de la capacité mnémonique de l'espace, ainsi que la manière dont les souvenirs sont influencés et retravaillés par la conscience. Pour Perec il est évident que l'image du passé gardée par la mémoire est susceptible d'être déformée au cours du temps. Ce genre de distorsion de la mémoire est, en effet, une conséquence de la superposition de l'image de la réalité perçue à un instant donné dans le temps avec notre état émotionnel de ce moment ${ }^{3}$. Cet effet du « vieillissement de la mémoire » est assez visible dans Je me souviens qui constitue un recueil de souvenirs quotidiens dont certains, comme l'affirme l'auteur, ont été significativement travestis.

Les principes établis pour la partie inédite de Lieux ont été adoptés dans une autre œuvre inachevée de l'auteur - Lieux où j'ai dormi - dont le but était de faire «l'inventaire aussi exhaustif et précis que possible» (PEREC, 2012:47) de tous les lieux où il avait dormi, y compris les voitures, les salles d'hôpital, etc. Ce faisant, Perec voulait écrire une sorte d'autobiographie spatiale, créée grâce à un énorme travail de remémoration. L'auteur s'est placé ainsi dans la position de «l'homme qui dort » et qui «tient en cercle autour de lui le fil des heures, l'ordre des années et des mondes » (PROUST, 2012:10). L'association à l'œuvre de Proust devient d'autant plus évidente lorsque Perec, en parlant de son programme littéraire, indique que l'espace de la chambre fonctionne chez lui exactement comme une madeleine proustienne (PEREC, 2012 : 47). L'objectif principal de Lieux où j'ai dormi, aussi bien que de la seconde étape de Lieux, se voulait donc une mise en œuvre de ce fonctionnement de la mémoire affective.

\footnotetext{
${ }^{3}$ Perec a consacré une partie de son travail littéraire proprement aux rapports entre le temps et le souvenir. Le sujet concernant les modifications des souvenirs fait partie du roman $W$ où le Souvenir d'enfance et des commentaires de l'auteur concernant son autre œuvre - Je me souviens. Cet aspect de l'écriture de Perec est également analysé par Philippe Lejeune dans La mémoire et l'oblique, op. cit. p. 75-76.
} 


\section{Palais de la mémoire}

Nous pouvons également noter que l'espace et la mémoire influent l'un sur l'autre de deux manières différentes. D'un côté, ce sont les souvenirs qui renvoient à un réservoir de perceptions subjectives et gardent une image émotionnelle concrète qui est identifiée à un lieu donné. Le déclenchement de la mémoire des lieux, tel qu'il se présente dans les textes perecquiens, permet de pénétrer encore une fois dans la réalité sensorielle d'un souvenir que nous gardons, tout à fait comme c'est le cas chez Proust qui parle, quant à lui, d'un «fauteuil magique » qui rend possible de « voyager à toute vitesse dans le temps et dans l'espace » (PROUST, 2012 : 10). Ce type de relation entre l'espace et le souvenir est d'ailleurs bien connu depuis l'Antiquité. Le « palais de la mémoire », appelé aussi « méthode des lieux », dont l'origine remonte à Simonide de Céos, constitue, en réalité, l'une des méthodes les plus efficaces et rapides de mémorisation, permettant d'enregistrer une grande quantité d'informations à l'aide de la mémoire spatiale ${ }^{4}$. L'idée qui se trouve à l'origine de Lieux ou j'ai dormi et de la partie du projet Lieux écrite à partir des associations des souvenirs, repose essentiellement sur cette technique, grâce à laquelle Perec a créé ce que nous pouvons considérer comme une poétique de l'anamnèse. Pourtant, dans ces deux cas la construction du «palais de la mémoire » perecquien prend différentes formes. Dans Lieux où j'ai dormi cette construction, à cause de son acuité extrême, ressemble plutôt à la première étape de Lieux, sauf que cette fois-ci la description spatiale ne résultait plus de l'observation sur place, mais uniquement du travail mnémonique. Le processus de l'écriture consistait alors à visualiser les lieux du passé, à les contempler, et ensuite à transcrire l'organisation des éléments ordonnés de l'image visualisée. Quant à la deuxième partie de Lieux, le mécanisme de la création était similaire, à la seule différence que la transcription finale était focalisée non pas sur l'apparence statique de l'espace, mais sur l'image affective qui y était ancrée.

D'un autre côté, nous pouvons observer que c'est l'espace lui-même qui porte aussi les empreintes de ses « usagers », qui est capable de révéler des tranches du passé et de mettre en marche des processus mnémoniques permettant d'atteindre les souvenirs dont nous ne réalisons même pas l'existence. Une telle conception de la mémoire spatiale se manifeste dans les lieux que Perec a choisis pour son œuvre. Il a commencé à visiter régulièrement la rue Vilin quand celle-ci ne ressemblait plus qu'aux vestiges d'une ancienne vie de l'avant-guerre. L'auteur était donc conscient que les empreintes imprimées dans l'espace de la rue, dans sa mémoire unique, allaient disparaître aussitôt que la rue cesserait d'exister. En disant que la ville d'origine de son père est « effacée », Perec a noté alors qu'il y était venu trop tard et que les lieux qu'il voulait y trouver avaient déjà été remplacés par de nouveaux espaces qui ont supprimé toutes les empreintes du passé.

\section{Espace - une interrogation continue}

En fin de compte, Perec se définit comme un « usager de l'espace » (PEREC, 2012 : page non numérotée), ce qui signifie, en d'autres termes, quelqu' un qui ne se

\footnotetext{
${ }^{4}$ Pour plus d'informations sur ce sujet, voir Yates, F. (1966). L'art de la mémoire, Paris, Gallimard.
} 
limite pas à une simple observation passive, mais qui marque cet espace de sa propre histoire. Comme l'indique Dominique Rabaté, "'l'usager de l'espace' est un individu parmi d'autres qui changent de lieux, qui rêvent d'une impossible patrie, et voyagent, de signes en signes. Lui le fait par l'écriture qui est peut-être la meilleure inscription possible, dans un espace à la fois mental et matériel » (RABATÉ, 2012 : 51). Par conséquent, si Perec tente d'entrer dans un espace, de se l'approprier, il le fait exactement à travers son écriture. Et écrire, selon Perec, c'est «laisser, quelque part, un sillon, une trace, une marque ou quelques signes » (PEREC, 2012: 180). Ainsi, il convient de noter que l'espace de la mémoire perecquienne n'enregistre pas seulement ce qui reste du passé des autres, mais elle devient aussi le lieu de la création de ses propres empreintes laissées dans les mots et dans les phrases à déchiffrer pour les autres. À travers son écriture, l'auteur établit enfin les lieux abstraits de ses pensées et souvenirs, mais, en même temps, ceux qui sont bien visibles et palpables. Les lieux perecquiens sont remplis de signes et de pages qui occupent les espaces réels de la bibliothèque - «le livre s'en va, en essayant de s'élargir : comme un caillou qu'on jette dans une rivière et qui fait des cercles » (PAWLIKOWSKA, $2003: 204)$.

Dans un entretien mené en 1979 par Frank Venaille, Perec a remarqué qu' « il y a trois aspects dans le travail sur la mémorisation. D'abord la quotidienneté complètement mise à plat, ensuite la recherche de [sa] propre histoire d'une manière traditionnelle et puis enfin cette mémoire fictionnelle » (PEREC, 1990: 86). Ce premier aspect - la mise à plat de la quotidienneté - se résume, bien évidemment, dans la poétique de l'infra-ordinaire, orientée vers une poursuite de petits morceaux de la vie, ceux qui coulent imperceptiblement entre les doigts. Ces particules de l'ordinaire perpétuées dans Lieux vont pourtant au-delà d'un ensemble de perceptions subjectives et s'inscrivent dans une image plus vaste de la mémoire collective. Dans Espèces d'espaces Perec se demande : «L'espace de notre vie n'est ni continu, ni infini, ni homogène, ni isotrope. Mais sait-on précisément où il se brise, où il se courbe, où il se déconnecte et où il se rassemble ?» (PEREC, 2012: page non numérotée). L'espace se veut donc une interrogation continue, mais c'est cette recherche, le questionnement sans réponse qui constitue l'essence de La rue Vilin et le caractérise comme le tombeau d'un amour. Comme le note Perec, l'espace reste toujours un doute avec ses fissures et incertitudes. Mais s'il existe une conquête de l'espace, c'est sans doute celle de l'écriture. Ici, la mémoire semble être la plus infaillible: enfermée dans le signe - le seul espace stable, intouché ou presque intouchable, le véritable point de départ.

\section{BIBLIOGRAPHIE}

Bellos David (1994), Georges Perec, une vie dans les mots: biographie, Paris, Editions du Seuil.

BLANCHOT Maurice (1969), L'entretien infini, Paris, Gallimard.

GETZLER Pierre ; DEPAULE, Jean-Charles (2012), Perec, la langue, la ville. Europe, $\mathrm{n}^{\circ}$ 993-994, p. 83-94.

HECK Maryline (2012), L' «infra-ordinaire », une poétique du regard. Europe, $\mathrm{n}^{\circ}$ 993-994, p. 62-70. 
LEJEUNE Philippe (1991), La mémoire et l'oblique, Paris, P.O.L.

PAWLIKOWSKA Ewa (2003), Entretien Georges Perec / Ewa Pawlikowska, in :

D. BERTELli ; M. RIBIÈRE (Eds.), Entretiens et Conférences, Volume II 19791981, Paris, Joseph K.

PEREC Georges (1990), Je suis né, Paris, Éditions du Seuil.

PEREC Georges (1989), L'infra-ordinaire, Paris, Editions du Seuil.

PEREC Georges (1985), Penser/classer, Paris, Hachette.

PEREC Georges (1975), Wou le Souvenir d'enfance, Paris, Denoël.

PROUST Marcel (2012), A la recherche du temps perdu, Paris, Editions Humanis.

RABATÉ Dominique (2012), Comme tout le monde, je suppose, Europe, no 993-994, 43-51.

RoY Claude (1983), Permis de séjour, 1977-1982, Paris, Gallimard. 